\title{
Editorial
}

\section{Invitation and Welcome to the 43rd Annual Meeting of the Society for Neuropediatrics}

\author{
Bernd A. Neubauer ${ }^{1}$ Andreas Hahn ${ }^{1}$ \\ ${ }^{1}$ Department of Pediatric Neurology, University Hospital Giessen, \\ Giessen, Germany
}

Neuropediatrics 2017;48:61.

It is with great pleasure that we invite you to join us at our $43^{\text {rd }}$ Annual Meeting of the Society for Neuropediatrics in Bad Nauheim. Bad Nauheim is a small art nouveau, spa and resort town in the vicinity of the Frankfurt central station and airport. Historically, the town has hosted prominent personalities such as Zar Nikolaus II, Albert Einstein, Franklin T. Roosevelt, and many others who sought and found recovery and recreation here.

The Scientific Committee has prepared an exciting program that will be presented by renowned speakers known to be experts in their field. Pediatric neurology is going through exciting times. Many of the diseases we are caring for are of genetic origin. In the area of diagnostic genomics and increasingly successful translational medicine, current clinical practice is changing at a fast pace. Metabolic and degenerative syndromes thought to be entirely resistant to causal treatment can now be influenced by biologicals that sprout from the laboratories and reach into the clinics at a high pace. The focus will be laid on therapies that are about to enter clinical practice within the next 1 or 2 years. This thread will include more frequent disorders, such as brain tumors, as well as orphan diseases. System disorders, bridging the gap from congenital brain malformations to multiorgan diseases such as the "ciliopathies," and new techniques in metabolic diagnostics will be mirrored.

However, our meeting is not restricted to molecular medicine by any means. The multiprofessional care for children growing to become adults with "coma vigile" as a lifelong burden and challenge to the caretakers will be covered comprehensively. A spectrum of new developments in functional rehabilitation medicine will receive an adequate focus in two separate sessions. The intersection of pediatric neurology and neuropsychology will be covered addressing "central visual perception disorders" in children. This is to name just some further examples.

A traditional and extremely successful stronghold of our annual meeting is the "Academy of Pediatric Neurology," a series of elaborate teaching and working group sessions, covering major topics in our field. Finally, we will try to draw a historical circle, shed some light on the beginnings of pediatric neurology in Germany, and end with a historic overview on the recognition and delineation of Rett Syndrome by a long time contemporary witness. Also, this year the "Desitin Investigator Award" will be presented by the laureate. This year Dr. Darius Ebrahimi-Fakhari will report on his price winning work entitled "Impaired Mitochondrial Dynamics and Mitophagy in Neuronal Models of Tuberous Sclerosis."

Our meeting will take place from April 27 to 29, 2017. The complete program can be downloaded at http://www.neuropaediatrie.com.

Traditionally, our society meetings have provided an excellent platform for the exchange of clinical and scientific knowledge on the latest developments and emerging challenges in our field. We look forward to welcoming you in Bad Nauheim in April 2017.
Address for correspondence

Bernd A. Neubauer, MD,

Department of Pediatric

Neurology, University Hospital

Giessen, 35395 Giessen,

Feulgenstrasse 12, Germany

(e-mail: Bernd.A.

Neubauer@paediat.med.uni-

giessen.de). (c) 2017 Georg Thieme Verlag KG

Stuttgart · New York
DOI http://dx.doi.org/ 10.1055/s-0037-1601319. ISSN 0174-304X. 